\title{
THE LOCAL SPECTRAL RADIUS OF A NONNEGATIVE ORBIT OF COMPACT LINEAR OPERATORS
}

\author{
MIHÁLY PITUK
}

\begin{abstract}
We consider orbits of compact linear operators in a real Banach space which are nonnegative with respect to the partial ordering induced by a given cone. The main result shows that under a mild additional assumption the local spectral radius of a nonnegative orbit is an eigenvalue of the operator with a positive eigenvector.
\end{abstract}

\section{INTRODUCTION AND THE MAIN RESULT}

Let $(X,\|\cdot\|)$ be a real Banach space. The symbol $\mathcal{B}(X)$ denotes the space of bounded linear operators in $X$ equipped with the operator norm. By the spectrum of $T \in \mathcal{B}(X)$, denoted by $\sigma(T)$, we mean the spectrum of the complex extension $T_{\mathbb{C}}: X_{\mathbb{C}} \rightarrow X_{\mathbb{C}}$, where $X_{\mathbb{C}}$ is the complexification of $X$, the set of formal pairs $x+i y$ with $x, y \in X$ equipped with the norm $\|x+i y\|=$ $\max _{0 \leq t \leq 2 \pi}\|(\sin t) x+(\cos t) y\|$, and $T_{\mathbb{C}}(x+i y)=T x+i T y$. A similar remark holds for the eigenvalues of $T \in \mathcal{B}(X)$. The set of eigenvalues, the so-called point spectrum of $T \in \mathcal{B}(X)$, is denoted by $\sigma_{p}(T)$. The spectral radius of $T \in \mathcal{B}(X)$ is defined by $r(T)=\max _{\lambda \in \sigma(T)}|\lambda|$. Note that according to Gelfand's formula

$$
r(T)=\lim _{n \rightarrow \infty} \sqrt[n]{\left\|T^{n}\right\|}
$$

for every $T \in \mathcal{B}(X)$. An operator $T \in \mathcal{B}(X)$ is called compact if the closure of $T(B)$ is compact, where $B$ denotes the unit ball in $X$. The space of compact operators in $X$ will be denoted by $\mathcal{K}(X)$. It is known that if $T \in \mathcal{K}(X)$, then every nonzero element of the spectrum $\sigma(T)$ is an eigenvalue of $T$. Hence

$$
r(T)=\max _{\lambda \in \sigma_{p}(T)}|\lambda| \quad \text { whenever } T \in \mathcal{K}(X) \text { and } r(T)>0 .
$$

A set $K \subset X$ is called a cone if conditions (i), (ii), and (iii) below hold.

(i) $K$ is a nonempty, convex and closed subset of $X$,

2010 Mathematics Subject Classification. Primary 47A11; Secondary 39A10.

Key words and phrases. compact linear operator; orbit; local spectral radius; cone; eigenvalue.

This work was supported by the Hungarian National Foundation for Scientific Research (OTKA) Grant No. K. 101217. 
(ii) $t K \subset K$ for all $t \geq 0$, where $t K=\{t x \mid x \in K\}$,

(iii) $K \cap(-K)=\{\theta\}$, where $-K=\{-x \mid x \in K\}$.

Let $K \subset X$ be a cone. Then $K$ induces a partial ordering $\leq_{K}$ on $X$ by $x \leq_{K} y$ if and only if $y-x \in K$. An element $x \in X$ is called $K$-nonnegative if $\theta \leq_{K} x$. An operator $T \in \mathcal{B}(X)$ is called $K$-nonnegative if $\theta \leq_{K} x$ implies $\theta \leq_{K} T x$. Clearly, $x \in X$ is $K$-nonnegative if and only if $x \in K$, and $T \in \mathcal{B}(X)$ is $K$-nonnegative if and only if $T(K) \subset K$. Recall that a cone $K \subset X$ is total if $\operatorname{cl}(K-K)=X$, where $\operatorname{cl}(K-K)$ denotes the closure of the set $K-K=\{x-y \mid x, y \in K\}$.

Krein and Rutman [5] proved the following result about the spectral radius of positive compact operators (see also [4] and [8] for secondary sources).

Theorem 1.1. Suppose that $(X,\|\cdot\|)$ is a real Banach space and $K \subset X$ is a total cone. Assume also that $T \in \mathcal{K}(X)$ is a $K$-nonnegative operator with $r(T)>0$. Then there exists $v \in K \backslash\{\theta\}$ such that $T v=r(T) v$ so that $r(T) \in$ $\sigma_{p}(T)$.

Note that in the finite dimensional case $X=\mathbb{R}^{n}$ and $K=\mathbb{R}_{+}^{n}$ Theorem 1.1 implies the Perron-Frobenius theorem stating that the spectral radius of a nonnegative $n \times n$ matrix $A$ is always an eigenvalue of $A$ with a nonnegative eigenvector (see, e.g., [1]).

In this paper we will prove an analogue of Theorem 1.1 for the local spectral radius corresponding to a nonnegative orbit of $T \in \mathcal{K}(X)$. By an orbit of $T \in \mathcal{B}(X)$, we mean a sequence $\left\{T^{n} x\right\}_{n=0}^{\infty}$, where $x \in X$ is a given vector. The local spectral radius of $T \in \mathcal{B}(X)$ at $x \in X$ is denoted by $\bar{r}(T ; x)$ and is defined by

$$
\bar{r}(T ; x)=\limsup _{n \rightarrow \infty} \sqrt[n]{\left\|T^{n} x\right\|} .
$$

Note that $\bar{r}(T ; x)$ describes the exponential growth of the orbit $\left\{T^{n} x\right\}_{n=0}^{\infty}$. Its logarithm is the Lyapunov exponent. Define also

$$
\underline{r}(T ; x)=\liminf _{n \rightarrow \infty} \sqrt[n]{\left\|T^{n} x\right\|} .
$$

Evidently, $\underline{r}(T ; x) \leq \bar{r}(T ; x)$. It is known that if $T$ is merely bounded, then the last inequality may be strict (see [6] for details and further related results). However, if $T$ is compact, then $\underline{r}(T ; x)=\bar{r}(T ; x)$ for all $x \in X$ and the following analogue of Conclusion (1.1) holds.

Theorem 1.2. Suppose that $(X,\|\cdot\|)$ is a real Banach space and $T \in \mathcal{K}(X)$. Then for every $x \in X$ the limit

$$
r(T ; x)=\lim _{n \rightarrow \infty} \sqrt[n]{\left\|T^{n} x\right\|}
$$

exists. Furthermore, if $r(T ; x)>0$ for some $x \in X$, then $r(T ; x)$ is the modulus of one of the eigenvalues of $T$, that is,

$$
r(T ; x)=|\lambda| \quad \text { for some } \lambda \in \sigma_{p}(T) .
$$


Theorem 1.2 follows from Corollary B.3 from Appendix B of [2] and from the Riesz-Schauder theory of compact linear operators.

Our main result is the following theorem.

Theorem 1.3. Suppose that $(X,\|\cdot\|)$ is a real Banach space, $K \subset X$ is a cone and $T \in \mathcal{K}(X)$. Assume also that for some $x \in X$ conditions

$$
T^{n} x \in K \quad \text { for all } n=0,1,2, \ldots
$$

and

$$
r(T ; x)>0
$$

hold. Then there exists $v \in K \backslash\{\theta\}$ such that $T v=r(T ; x) v$ so that $r(T ; x) \in$ $\sigma_{p}(T)$.

Note that in contrast with Theorem 1.1, in Theorem 1.3 we do not require the $K$-nonnegativity of operator $T$. We assume merely that the orbit $\left\{T^{n} x\right\}_{n=0}^{\infty}$ is $K$-nonnegative (see (1.5)). For the finite dimensional analogue of Theorem 1.3 for Poincaré difference equations, see [7].

\section{ProOF}

Before we present the proof of Theorem 1.3, we recall some facts from the spectral theory of bounded linear operators (see Chapter VII of [3]) and we establish some lemmas.

Suppose that $(X,\|\cdot\|)$ is a complex Banach space and $T \in \mathcal{B}(X)$. Let $\sigma$ be a closed isolated subset of the spectrum $\sigma(T)$. The spectral projection (Riesz idempotent) of $T$ corresponding to $\sigma$ is denoted by $P_{\sigma}$ and is defined by

$$
P_{\sigma}=\frac{1}{2 \pi i} \int_{\Gamma}(z-T)^{-1} d z,
$$

where $\Gamma$ is any positively oriented Jordan system such that

$$
\sigma \subset \operatorname{ins} \Gamma \quad \text { and } \quad \sigma(T) \backslash \sigma \subset \text { out } \Gamma,
$$

where ins $\Gamma$ and out $\Gamma$ denote the inside of $\Gamma$ and the outside of $\Gamma$, respectively (see Chapter VII, Section 6.9 of [3]). The bounded linear operator $P_{\sigma}$ has properties

$$
P_{\sigma}^{2}=P_{\sigma}
$$

and

$$
P_{\sigma} T=T P_{\sigma}
$$

The set $P_{\sigma}(X)$ is called the generalized eigenspace of $T$ corresponding to $\sigma$. It follows from (2.3) that $T$ maps $P_{\sigma}(X)$ into itself and if $T_{\sigma}=\left.T\right|_{P_{\sigma}(X)}$, the restriction of $T$ onto $P_{\sigma}(X)$, then the spectrum of $T_{\sigma}: P_{\sigma}(X) \rightarrow P_{\sigma}(X)$ is $\sigma$, that is,

$$
\sigma\left(T_{\sigma}\right)=\sigma .
$$


Assume in addition that $T \in \mathcal{K}(X)$, i.e. $T$ is compact. Then every $\lambda \in \sigma(T) \backslash\{0\}$ is an eigenvalue of $T$ and for every $\epsilon>0$ the set $\{\lambda \in \sigma(T)|| \lambda \mid \geq \epsilon\} \subset \sigma_{p}(T)$ is finite. It is known that each $\lambda \in \sigma(T) \backslash\{0\}$ is a pole of the resolvent $(z-T)^{-1}$ and the generalized eigenspace $P_{\{\lambda\}}(X)$ is finite dimensional (see Corollary 7.8 of [3]). Now suppose that $\sigma \subset \sigma(T) \backslash\{0\}$ is a finite set. By the Residue Theorem, we have

$$
P_{\sigma}=\sum_{\lambda \in \sigma} P_{\{\lambda\}}
$$

and therefore the generalized eigenspace $P_{\sigma}(X)$ is also finite dimensional. Furthermore, since $0 \notin \sigma$, in this case operator $T_{\sigma}: P_{\sigma}(X) \rightarrow P_{\sigma}(X)$ is invertible.

As noted on p. 799 of [8], if $T \in \mathcal{B}(X)$ and

$$
\sigma(T)=\sigma_{1} \cup \sigma_{2} \cup \cdots \cup \sigma_{k},
$$

where $\sigma_{1}, \sigma_{2}, \ldots, \sigma_{k}$, are pairwise disjoint closed isolated subsets of $\sigma(T)$, then $X$ can be decomposed into the direct sum

$$
X=P_{\sigma_{1}}(X) \oplus P_{\sigma_{2}}(X) \oplus \cdots \oplus P_{\sigma_{k}}(X) .
$$

If $(X,\|\cdot\|)$ is a real Banach space and $T \in \mathcal{B}(X)$, then the spectral projection $P_{\sigma}$ can be defined as in $(2.1)$ for the complex extension $T_{\mathbb{C}}$. If we consider only sets $\sigma \subset \sigma(T)$ which are symmetric with respect to the real axis, then the restriction of $P_{\sigma}$ onto $X$ is a projection operator on $X$ and the set $P_{\sigma}(X)$ is called the generalized real eigenspace of $T$ corresponding to $\sigma$. Finally, if each $\sigma_{j}, 1 \leq j \leq k$, in (2.5) is symmetric with respect to the real axis, then the splitting result (2.6) remains valid for real Banach spaces.

Now we establish a lemma will play an important role in the proof of Theorem 1.3.

Lemma 2.1. Let $(X,\|\cdot\|)$ be a real Banach space and $T \in \mathcal{K}(X)$. Suppose that for some $x \in X$ conditions (1.5) and (1.6) hold. Define

$$
\begin{gathered}
\sigma_{-}=\{\lambda \in \sigma(T)|| \lambda \mid<r(T ; x)\}, \\
\sigma_{0}=\{\lambda \in \sigma(T)|| \lambda \mid=r(T ; x)\},
\end{gathered}
$$

and

so that

$$
\sigma_{+}=\{\lambda \in \sigma(T)|| \lambda \mid>r(T ; x)\}
$$

and

$$
\sigma(T)=\sigma_{-} \cup \sigma_{0} \cup \sigma_{+}
$$

$$
X=P_{\sigma_{-}}(X) \oplus P_{\sigma_{0}}(X) \oplus P_{\sigma_{+}}(X) .
$$

Let

$$
K_{0}=K \cap P_{\sigma_{0}}(X) .
$$

Then there exists $x_{0} \in K_{0}$ with $\left\|x_{0}\right\|=1$ such that

$$
T^{n} x_{0} \in K_{0} \quad \text { for all } n=0,1,2, \ldots
$$


The proof of Lemma 2.1 will be based on the following simple result.

Lemma 2.2. Let $(X,\|\cdot\|)$ be a Banach space and $T \in \mathcal{B}(X)$. Then for every $x \in X$, we have

$$
\bar{r}(T ; x) \leq \max _{\lambda \in \sigma(T)}|\lambda| .
$$

If $\operatorname{dim} X<\infty$ and $T \in \mathcal{B}(X)$ is invertible, then for every $x \in X \backslash\{\theta\}$, we have

$$
r(T ; x) \geq \min _{\lambda \in \sigma(T)}|\lambda|>0 .
$$

Proof. Conclusion (2.14) follows immediately from the obvious inequality

$$
\left\|T^{n} x\right\| \leq\left\|T^{n}\right\|\|x\|, \quad x \in X, n \geq 0,
$$

and Gelfand's spectral radius formula.

Suppose that $\operatorname{dim} X<\infty$ and $T \in \mathcal{B}(X)$ is invertible. Then $\sigma(T)=\sigma_{p}(T)$, $0 \notin \sigma(T)$, and the spectrum of $T^{-1}$, the inverse of $T$, is given by

$$
\sigma\left(T^{-1}\right)=\left\{\lambda^{-1} \mid \lambda \in \sigma(T)\right\} .
$$

Hence

$$
r\left(T^{-1}\right)=\max _{\lambda \in \sigma(T)}|\lambda|^{-1}=\frac{1}{\min _{\lambda \in \sigma(T)}|\lambda|} .
$$

This, combined with Gelfand's spectral radius formula, yields

$$
\lim _{n \rightarrow \infty} \sqrt[n]{\left\|T^{-n}\right\|}=\frac{1}{\min _{\lambda \in \sigma(T)}|\lambda|},
$$

where $T^{-n}=\left(T^{-1}\right)^{n}$ for $n \geq 0$. Choose $\epsilon>0$. The previous limit relation implies

$$
\left\|T^{-n}\right\|<\left(\frac{1}{\min _{\lambda \in \sigma(T)}|\lambda|}+\epsilon\right)^{n}
$$

for all large $n$. This, together with the inequality

$$
\|x\|=\left\|T^{-n} T^{n} x\right\| \leq\left\|T^{-n}\right\|\left\|T^{n} x\right\|, \quad x \in X, n \geq 0,
$$

implies

$$
\left\|T^{n} x\right\| \geq \frac{1}{\left\|T^{-n}\right\|}\|x\| \geq\left(\frac{1}{\min _{\lambda \in \sigma(T)}|\lambda|}+\epsilon\right)^{-n}\|x\|
$$

for all $x \in X$ and $n$ sufficiently large. The last inequality implies that if $x \in$ $X \backslash\{\theta\}$, then

$$
r(T ; x) \geq\left(\frac{1}{\min _{\lambda \in \sigma(T)}|\lambda|}+\epsilon\right)^{-1} .
$$

(The existence of the limit $r(T ; x)$ (see (1.4)) follows from Theorem 1.2 since every linear operator in a finite dimensional Banach space is compact.) Letting $\epsilon \rightarrow 0$ in the last inequality, we obtain (2.15). 
Remark 1. The result of Lemma 2.2 is not really new. Conclusion (2.15) is in fact a corollary of Theorem 1.2 of [7]. We have included the above proof only for the readers' convenience.

Now we give a proof of Lemma 2.1 .

Proof of Lemma 2.1. Define

$$
y_{n}=\frac{T^{n} x}{\left\|T^{n} x\right\|}, \quad n \geq 0 .
$$

By virtue of (1.6), the sequence $\left\{y_{n}\right\}_{n=0}^{\infty}$ is well-defined. Clearly, $\left\|y_{n}\right\|=1$ for all $n \geq 0$ and hence

$$
\left\|P_{\sigma_{0}} y_{n}\right\| \leq\left\|P_{\sigma_{0}}\right\|\left\|y_{n}\right\|=\left\|P_{\sigma_{0}}\right\|, \quad n \geq 0 .
$$

This shows that $\left\{P_{\sigma_{0}} y_{n}\right\}_{n=0}^{\infty}$ is a bounded sequence in the generalized eigenspace $P_{\sigma_{0}}(X)$. Since $T$ is compact and $r(T ; x)>0$, the set $\sigma_{0} \subset \sigma(T) \backslash\{0\}$ is finite. As noted before, this implies that $\operatorname{dim} P_{\sigma_{0}}(X)<\infty$. Therefore the bounded sequence $\left\{P_{\sigma_{0}} y_{n}\right\}_{n=0}^{\infty}$ has a convergent subsequence. Consequently, there exist $x_{0} \in P_{\sigma_{0}}(X)$ and a sequence $n_{k} \rightarrow \infty$ as $k \rightarrow \infty$ such that

$$
\lim _{k \rightarrow \infty} P_{\sigma_{0}} y_{n_{k}}=x_{0}
$$

We claim that

$$
\lim _{k \rightarrow \infty} y_{n_{k}}=x_{0}
$$

In view of (2.17) and the relation

$$
y_{n_{k}}=P_{\sigma_{-}} y_{n_{k}}+P_{\sigma_{0}} y_{n_{k}}+P_{\sigma_{+}} y_{n_{k}}, \quad k \geq 0
$$

in order to prove (2.18), it is enough to show that

$$
\lim _{n \rightarrow \infty} P_{\sigma_{-}} y_{n}=\theta
$$

and

$$
\lim _{n \rightarrow \infty} P_{\sigma_{+}} y_{n}=\theta .
$$

By virtue of (2.3) and (2.16), we have for $n \geq 0$,

$$
P_{\sigma_{-}} y_{n}=\frac{T^{n} P_{\sigma_{-}} x}{\left\|T^{n} x\right\|}=\frac{T_{\sigma_{-}}^{n} P_{\sigma_{-}} x}{\left\|T^{n} x\right\|},
$$

where $T_{\sigma_{-}}=\left.T\right|_{P_{\sigma_{-}}(X)}$. This, together with (1.2) and (1.4), implies

$$
\limsup _{n \rightarrow \infty} \sqrt[n]{\left\|P_{\sigma_{-}} y_{n}\right\|}=\limsup _{n \rightarrow \infty} \frac{\sqrt[n]{\left\|T_{\sigma_{-}}^{n} P_{\sigma_{-}} x\right\|}}{\sqrt[n]{\left\|T^{n} x\right\|}}=\frac{\bar{r}\left(T_{\sigma_{-}} ; P_{\sigma_{-}} x\right)}{r(T ; x)} .
$$

From Conclusion (2.14) of Lemma 2.2 and conditions (2.4) and (2.7), we obtain

$$
\bar{r}\left(T_{\sigma_{-}} ; P_{\sigma_{-}} x\right) \leq \max _{\lambda \in \sigma\left(T_{\sigma_{-}}\right)}|\lambda|=\max _{\lambda \in \sigma_{-}}|\lambda|<r(T ; x) .
$$


The last inequality implies that the limsup in (2.21) is less than one. Therefore $P_{\sigma_{-}} y_{n} \rightarrow \theta$ exponentially as $n \rightarrow 0$ and hence (2.19) holds.

Now we prove $(2.20)$. First we show that $P_{\sigma_{+}} x=\theta$. Suppose by way of contradiction that $P_{\sigma_{+}} x \neq \theta$. We have for $n \geq 0$,

$$
P_{\sigma_{+}} y_{n}=\frac{T^{n} P_{\sigma_{+}} x}{\left\|T^{n} x\right\|}=\frac{T_{\sigma_{+}}^{n} P_{\sigma_{+}} x}{\left\|T^{n} x\right\|},
$$

where $T_{\sigma_{+}}=\left.T\right|_{P_{\sigma_{+}}(X)}$. The compactness of $T$ and the definition of $\sigma_{+}$imply that $\sigma\left(T_{\sigma_{+}}\right)=\sigma_{+} \subset \sigma(T) \backslash\{0\}$ is a finite set. Consequently, the generalized eigenspace $P_{\sigma_{+}}(X)$ is finite dimensional and $T_{\sigma_{+}}: P_{\sigma_{+}}(X) \rightarrow P_{\sigma_{+}}(X)$ is invertible and compact. From (2.22), by the application of Theorem 1.2, we find that

$$
\lim _{n \rightarrow \infty} \sqrt[n]{\left\|P_{\sigma_{+}} y_{n}\right\|}=\lim _{n \rightarrow \infty} \frac{\sqrt[n]{\left\|T_{\sigma_{+}}^{n} P_{\sigma_{+}} x\right\|}}{\sqrt[n]{\left\|T^{n} x\right\|}}=\frac{r\left(T_{\sigma_{+}} ; P_{\sigma_{+}} x\right)}{r(T ; x)} .
$$

Since $P_{\sigma_{+}} x \neq \theta$, Conclusion (2.15) of Lemma 2.2 and (2.9) imply that

$$
r\left(T_{\sigma_{+}} ; P_{\sigma_{+}} x\right) \geq \min _{\lambda \in \sigma\left(T_{\sigma_{+}}\right)}|\lambda|=\min _{\lambda \in \sigma_{+}}|\lambda|>r(T ; x) .
$$

Hence the limit in (2.23) is greater than one. This implies that $\left\|P_{\sigma_{+}} y_{n}\right\| \rightarrow \infty$ exponentially as $n \rightarrow \infty$ contradicting the fact that

$$
\left\|P_{\sigma_{+}} y_{n}\right\| \leq\left\|P_{\sigma_{+}}\right\|\left\|y_{n}\right\|=\left\|P_{\sigma_{+}}\right\|, \quad n \geq 0 .
$$

Thus, $P_{\sigma_{+}} x=\theta$ which implies that

$$
P_{\sigma_{+}} y_{n}=\frac{T^{n} P_{\sigma_{+}} x}{\left\|T^{n} x\right\|}=\theta, \quad n \geq 0 .
$$

Hence (2.20) holds. As noted before, this completes the proof of (2.18).

By virtue of (2.16) and (2.18), we have

$$
\left\|x_{0}\right\|=\lim _{k \rightarrow \infty}\left\|y_{n_{k}}\right\|=1 \text {. }
$$

As shown before, $x_{0} \in P_{\sigma_{0}}(X)$. Further, $T$ and hence each $T^{n}, n \geq 0$, maps $P_{\sigma_{0}}(X)$ into itself. Consequently, $T^{n}\left(x_{0}\right) \in P_{\sigma_{0}}(X)$ for every $n \geq 0$. Thus, in order to prove $(2.13)$, it remains to show that

$$
T^{n} x_{0} \in K \quad \text { for every nonnegative integer } n \text {. }
$$

Let $n$ be a fixed nonnegative integer. By virtue of (2.16), (2.18), the continuity of $T$ and hence of $T^{n}$, we have

$$
T^{n}\left(x_{0}\right)=T^{n}\left(\lim _{k \rightarrow \infty} y_{n_{k}}\right)=\lim _{k \rightarrow \infty} T^{n} y_{n_{k}}=\lim _{k \rightarrow \infty} \frac{T^{n+n_{k}} x}{\left\|T^{n_{k}} x\right\|} .
$$


From assumption (1.5) and the cone property (ii), it follows that

$$
\frac{T^{n+n_{k}} x}{\left\|T^{n_{k}} x\right\|} \in K \quad \text { for every } k \geq 0 \text {. }
$$

From this, taking into account that $K$ is a closed set, we see that $T^{n}\left(x_{0}\right)$ as a limit of the above sequence from $K$ also belongs to $K$. Since $n \geq 0$ was arbitrary, this proves $(2.24)$.

Now we are in a position to give a proof of Theorem 1.3.

Proof of Theorem 1.3. Adopt the notation of Lemma 2.1. Define

$$
C=\left\{x \in K_{0} \mid\|x\| \leq 1 \text { and } T^{n} x \in K_{0} \text { for all } n=0,1, \ldots\right\}
$$

with $K_{0}$ as in (2.12). By the application of Lemma 2.1, we conclude that there exists $x_{0} \in C$ such that $\left\|x_{0}\right\|=1$. It is easily verified that $C$ is a convex closed subset of $P_{\sigma_{0}}(X)$. As noted in the proof of Lemma 2.1, the subspace $P_{\sigma_{0}}(X)$ is finite dimensional and if $T_{\sigma_{0}}=\left.T\right|_{P_{\sigma_{0}}(X)}$, then $T_{\sigma_{0}}: P_{\sigma_{0}}(X) \rightarrow P_{\sigma_{0}}(X)$ is invertible.

Define an operator $F: C \rightarrow P_{\sigma_{0}}(X)$ by

$$
F(x)=\frac{(1-\|x\|) x_{0}+\|x\| T_{\sigma_{0}} x}{\left\|(1-\|x\|) x_{0}+\right\| x\left\|T_{\sigma_{0}} x\right\|}, \quad x \in C .
$$

The cone property (iii), the fact that $x_{0} \neq \theta$ and the invertibility of operator $T_{\sigma_{0}}: P_{\sigma_{0}}(X) \rightarrow P_{\sigma_{0}}(X)$ imply that $F$ is well-defined. Clearly, $F$ is continuous on $C$. Further, the definition of $C$ and the cone properties (i) and (ii) imply that $F(C) \subset C$. By Brouwer's fixed point principle, there exists $v \in C$ such that $F(v)=v$. Since $\|v\|=\|F(v)\|=1$, it follows that $T_{\sigma_{0}} v=\rho v$, where $\rho=\left\|T_{\sigma_{0}}(v)\right\|$. Thus, $\rho$ is a nonnegative eigenvalue of $T_{\sigma_{0}}: P_{\sigma_{0}}(X) \rightarrow P_{\sigma_{0}}(X)$. Since the spectrum of $T_{\sigma_{0}}$ coincides with $\sigma_{0}$, we have $\rho=|\rho|=r(T ; x)$. Thus, $r(T ; x)$ is an eigenvalue of $T$ with eigenvector $v \in K$.

Remark 2. Under the hypotheses of Theorem 1.3, the set $K_{0}$ defined by $(2.12)$ is a cone in the finite-dimensional space $P_{\sigma_{0}}(X)$. Consequently, Theorem 1.3 can also be deduced from Conclusion (2.13) of Lemma 2.1 and Theorem 1.3 of [7]. Note that the above short proof is independent of Theorem 1.3 of [7]. It uses only Brouwer's fixed point theorem.

\section{REFERENCES}

[1] BERMAN, A.-PLEMMONS, R.: Nonnegative Matrices in the Mathematical Sciences, Academic Press, New York, 1979.

[2] CHEN, M.-CHEN, X.-Y.-HALE, J. K.: Structural stability for time-periodic onedimensional parabolic equations, J. Differential Equations 96 (1992), 355-418.

[3] CONWAY, J. B.: A Course in Functional Analysis. Second Edition, Springer-Verlag, New York, 1990. 
[4] KRAsnoselskiJ, M. A.-LIfShits, JE. A.-SOBOlEV, A. V.: Positive Linear Systems. The Method of Positive Operators, Heldermann Verlag, Berlin, 1989.

[5] KREIN, M. G.-RUTMAN, M. A.: Linear operators leaving a cone invariant in a Banach space, Uspekhi Mat. Nauk 3 no.1 (23) (1948), 3-95 (in Russian); English transl.: Amer. Math. Soc. Transl. 26 (1950).

[6] MÜLLER, V.: Spectral Theory of Linear Operators and Spectral System in Banach Algebras. Second Edition, Birkhäuser, Basel, 2007.

[7] OBAYA, R.-PITUK, M.: A variant of the Krein-Rutman theorem for Poincaré difference equations, J. Difference Equa. Appl. 18 (2012), 1751-1762.

[8] ZEIDLER, E.: Nonlinear Functional Analysis and its Applications I. Fixed-Point Theorems, Springer-Verlag, New York, 1986.

Department of Mathematics

UnIVERSITY OF PANNONIA

P.O. Box 158

H-8201 VESZPRÉM

HUNGARY

E-mail address: pitukm@almos.uni-pannon.hu 